\title{
On the numerical evaluation of scalar two-loop self-energy diagrams
}

\author{
F.A. Berends * \\ Instituut-Lorentz, University of Leiden, \\ P.O.B. 9506, 2300 RA Leiden, The Netherlands \\ J.B. Tausk ${ }^{\dagger}$ \\ Department of Physics, \\ University of Durham, Durham, DH1 3LE, England.
}

January 1994

\begin{abstract}
In this paper a method is presented for the numerical evaluation of scalar two-loop selfenergy diagrams. It is an extension of Kreimer's two-dimensional integral representation for convergent scalar integrals to the general case where divergencies occur. Where possible, the method is extensively compared with alternative techniques. From this it can be concluded, that the described two-dimensional numerical integration leads to a practical numerical answer for scalar two-loop self-energy diagrams with arbitrary masses.
\end{abstract}

*E-mail: berends@rulgm0.leidenuniv.nl

${ }^{\dagger}$ Research supported by the Stichting FOM. E-mail: J.B.Tausk@durham.ac.uk 


\section{Introduction}

With the ongoing precision experiments testing the electroweak theory there will be a growing necessity to perform second order radiative corrections. For the QED part of the corrections many useful results have been obtained for the line-shape and Bhabha scattering [1]. For the weak part some interesting results on the top mass dependence exist which have to do with the evaluation of self-energies in cases where some masses are considered very large and others negligible [2].

Eventually one would like to know the vector boson self-energies in two loops without such approximations. For this one needs several building blocks. One is the reduction of the self-energy two-loop integrals to a set of scalar integrals corresponding to scalar diagrams. This has been given in [3]. Another building block consists of the scalar integrals themselves. These scalar integrals can contain many mass combinations since the electroweak theory possesses a large number of different masses. It is known that many of these scalar diagrams cannot be calculated analytically, since the integrals are not expressible in terms of known functions such as polylogarithms.

Recently, there has been progress in the evaluation of two-loop scalar integrals in three respects.

The first achievement we want to mention concerns the numerical evaluation of the only two-loop scalar diagram which is convergent. A simple algorithm has been proposed [4] which involves a twofold numerical integration.

The second development is the derivation of small and large $p^{2}$ expansions for scalar two-loop diagrams $[5,6,7,8]$. The expansion coefficients have been obtained analytically for diagrams with arbitrary mass combinations. Moreover also for divergent diagrams these expansions have been obtained when the dimensional regularization scheme is used.

The third point is that analytical results have recently been obtained for a large number of mass combinations [9]. These results are both for divergent and convergent integrals.

Thanks to these developments it has now become possible to make a synthesis in the form of a numerical evaluation of all two-loop scalar diagrams, ultraviolet divergent or convergent. Thus the purpose of this paper is to supply a means to calculate any twoloop scalar self-energy diagram. The result will consist of pole terms in $\delta=\frac{4-D}{2}$ and a numerically evaluated finite part. A computer program based on this strategy has been made and tested against many results obtained in different ways i.e. by expansions or by analytical evaluation.

The actual outline of this paper is as follows. In section 2 we make an inventory of the two-loop scalar diagrams. In section 3 the numerical algorithm of ref. [4] is extended from a special convergent case to all cases, ultraviolet divergent or convergent. Comparisons between the calculations of this paper and other calculations, analytical or by expansions, are made in section 4 . For this some new analytical results are presented. Finally, our conclusions are given in section 5 .

\section{Inventory}

In this section we list the scalar two-loop diagrams, indicate whether they are analytically calculable and comment on the divergencies.

The basic two-loop self-energy diagrams are shown in Figs. 1 and 2. The notation for these diagrams is the same as in refs. [3, 9]. 


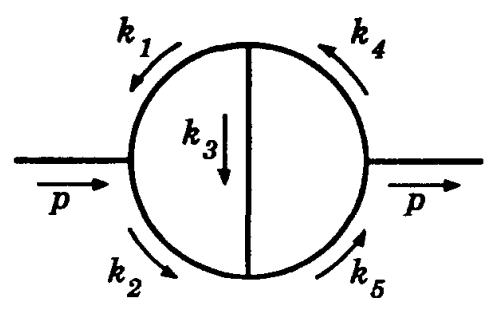

Figure 1: $T_{12345}\left(p^{2} ; m_{1}^{2}, m_{2}^{2}, m_{3}^{2}, m_{4}^{2}, m_{5}^{2}\right)$

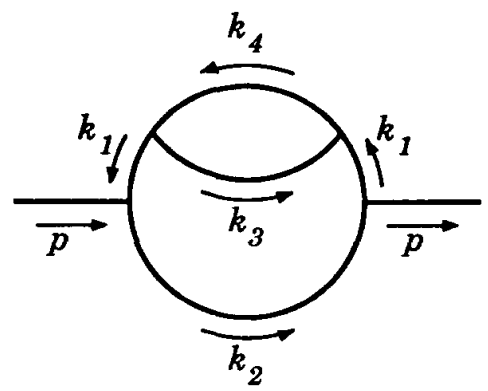

Figure 2: $T_{11234}\left(p^{2} ; m_{1}^{2}, m_{1}^{2}, m_{2}^{2}, m_{3}^{2}, m_{4}^{2}\right)$

The definition of the scalar integrals can be seen from the following specific case

$$
T_{12345}\left(p^{2} ; m_{1}^{2}, m_{2}^{2}, m_{3}^{2}, m_{4}^{2}, m_{5}^{2}\right)=\left\langle\left\langle\frac{1}{\left[k_{1}^{2}-m_{1}^{2}\right] \ldots\left[k_{5}^{2}-m_{5}^{2}\right]}\right\rangle\right\rangle,
$$

with

$$
\langle\langle\ldots\rangle\rangle=\int \frac{d^{D} q_{1}}{i \pi^{2}(2 \pi \mu)^{D-4}} \int \frac{d^{D} q_{2}}{i \pi^{2}(2 \pi \mu)^{D-4}}(\ldots),
$$

$D=4-2 \delta$ the dimension of space-time, and $\mu$ an arbitrary reference mass. The momenta $k_{i}$ of the propagators will be expressed in terms of the loop momenta $q_{1}$ and $q_{2}$ and the external momentum $p$. It should be noted that in $T_{11234}$ only the case is considered where the two propagators carrying the momentum $k_{1}$ also have the same mass. The reason is that for the unequal mass case the product of these two propagators can be written as the difference of the two propagators. Therefore the diagram is the difference of two diagrams with one $k_{1}$ propagator removed.

This particular diagram and a number of other diagrams which arise by removing propagators in Figs. 1 and 2 are given in Figs. 3 and 4.

The reducible diagrams will not be considered any further, since they are products of well-known one-loop integrals.

The functions $T_{134}$ and $T_{1134}$ are vacuum integrals and are known analytically for arbitrary masses. For completeness, they are given in the appendix. On the other hand the true two-point functions $T_{234}, T_{1234}, T_{11234}$ and $T_{12345}$ are only known for a number of special mass cases. In fact, it has been shown [10] that, for instance, $T_{12345}$ cannot be expressed in terms of polylogarithms if either of the following quantities is not zero:

$$
m_{1}^{2} m_{3}^{2} m_{5}^{2} p^{2} \prod_{+,-}\left(p^{2}-\left(m_{1} \pm m_{3} \pm m_{5}\right)^{2}\right)
$$




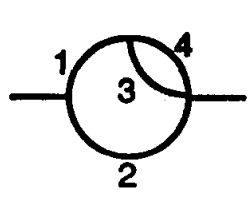

$T_{1234}$

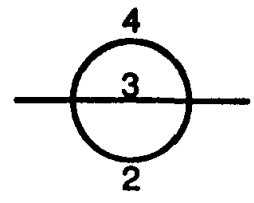

$T_{234}$

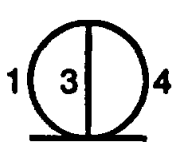

$T_{134}$

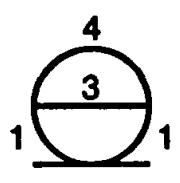

$T_{1134}$

Figure 3: Irreducible diagrams

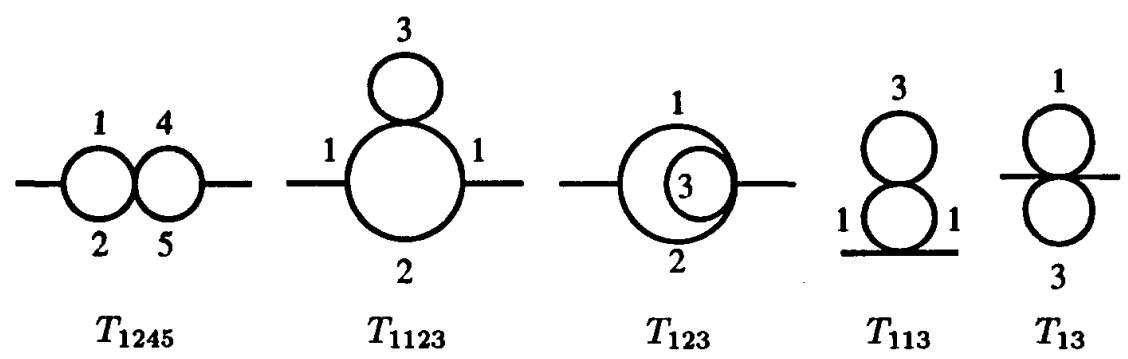

Figure 4: Reducible diagrams

$$
m_{2}^{2} m_{3}^{2} m_{4}^{2} p^{2} \prod_{+,-}\left(p^{2}-\left(m_{2} \pm m_{3} \pm m_{4}\right)^{2}\right) .
$$

The product is over all four combinations of signs.

Concerning the convergence of these integrals, we note that $T_{12345}$ is convergent, $T_{11234}$ has an ultraviolet divergent subdiagram giving rise to a $\delta^{-1}$ pole, and $T_{1234}$ and $T_{234}$ have subdivergences and overall divergences, which appear as $\delta^{-2}$ and $\delta^{-1}$ poles.

For general values of $p^{2}$ we can take the limits $m_{i} \rightarrow 0$ in $T_{12345}, T_{11234}, T_{1234}$ and $T_{234}$ as long as $m_{1} \neq 0$ in $T_{11234}$. The coefficients of $\delta^{-2}, \delta^{-1}$ and $\delta^{0}$ all have a regular behaviour when the masses vanish.

The diagram $T_{11234}$ becomes infrared divergent for all values of $p^{2}$ when $m_{1}=0$. For special values of $p^{2}$ and the masses infrared divergences may also occur in other diagrams.

\section{The algorithm}

Each of the integrals $T_{234}, T_{1234}$ and $T_{11234}$ is ultraviolet divergent. In order to calculate them numerically, we shall decompose them into two terms:

$$
T=T_{N}+T_{A} .
$$

This decomposition must satisfy two conditions. Firstly, the numerical part $T_{N}$ must be finite, so that it can be calculated by numerical integration. Secondly, the analytical part $T_{A}$ must be a combination of integrals for which analytical expressions are known.

To find a suitable decomposition, we use the following identity:

$$
\frac{1}{k^{2}-m^{2}}=\frac{1}{k^{2}}+\frac{m^{2}}{k^{2}\left(k^{2}-m^{2}\right)} \text {. }
$$


Applying this to propagators 3 and 4 in $T_{234}$, we obtain

$$
\begin{aligned}
T_{234}\left(p^{2} ; m_{2}^{2}, m_{3}^{2}, m_{4}^{2}\right)= & -T_{234}\left(p^{2} ; m_{2}^{2}, 0,0\right) \\
& +T_{234}\left(p^{2} ; m_{2}^{2}, m_{3}^{2}, 0\right)+T_{234}\left(p^{2} ; m_{2}^{2}, 0, m_{4}^{2}\right) \\
& +m_{3}^{2} m_{4}^{2} T_{23344}\left(p^{2} ; m_{2}^{2}, m_{3}^{2}, 0, m_{4}^{2}, 0\right) .
\end{aligned}
$$

The first three terms on the right hand side of this equation are all known analytically. The last term is finite, due to the extra propagators it contains. Therefore, both requirements mentioned above are satisfied if we define

$$
\begin{aligned}
T_{234 A}\left(p^{2} ; m_{2}^{2}, m_{3}^{2}, m_{4}^{2}\right)= & -T_{234}\left(p^{2} ; m_{2}^{2}, 0,0\right) \\
& +T_{234}\left(p^{2} ; m_{2}^{2}, m_{3}^{2}, 0\right)+T_{234}\left(p^{2} ; m_{2}^{2}, 0, m_{4}^{2}\right), \\
T_{234 N}\left(p^{2} ; m_{2}^{2}, m_{3}^{2}, m_{4}^{2}\right)= & m_{3}^{2} m_{4}^{2} T_{23344}\left(p^{2} ; m_{2}^{2}, m_{3}^{2}, 0, m_{4}^{2}, 0\right) .
\end{aligned}
$$

In a similar way, the following identities for $T_{1234}$ and $T_{11234}$ can be derived:

$$
\begin{aligned}
& T_{1234}\left(p^{2} ; m_{1}^{2}, m_{2}^{2}, m_{3}^{2}, m_{4}^{2}\right)=T_{1234}\left(p^{2} ; m_{1}^{2}, m_{2}^{2}, 0,0\right) \\
& \quad+m_{3}^{2} T_{12334}\left(p^{2} ; m_{1}^{2}, m_{2}^{2}, m_{3}^{2}, 0,0\right)+m_{4}^{2} T_{12344}\left(p^{2} ; m_{1}^{2}, m_{2}^{2}, 0, m_{4}^{2}, 0\right) \\
& \quad+m_{3}^{2} m_{4}^{2} T_{123344}\left(p^{2} ; m_{1}^{2}, m_{2}^{2}, m_{3}^{2}, 0, m_{4}^{2}, 0\right)
\end{aligned}
$$

and

$$
\begin{aligned}
& T_{11234}\left(p^{2} ; m_{1}^{2}, m_{1}^{2}, m_{2}^{2}, m_{3}^{2}, m_{4}^{2}\right)=T_{11234}\left(p^{2} ; m_{1}^{2}, m_{1}^{2}, m_{2}^{2}, 0,0\right) \\
& \quad+m_{3}^{2} T_{112334}\left(p^{2} ; m_{1}^{2}, m_{1}^{2}, m_{2}^{2}, m_{3}^{2}, 0,0\right)+m_{4}^{2} T_{112344}\left(p^{2} ; m_{1}^{2}, m_{1}^{2}, m_{2}^{2}, 0, m_{4}^{2}, 0\right) \\
& \quad+m_{3}^{2} m_{4}^{2} T_{1123344}\left(p^{2} ; m_{1}^{2}, m_{1}^{2}, m_{2}^{2}, m_{3}^{2}, 0, m_{4}^{2}, 0\right) .
\end{aligned}
$$

The first terms on the right hand sides of eqs. (10) and (11) are known analytically and can be found in ref. [9]. All the other terms are convergent. This leads us to define

$$
\begin{aligned}
T_{1234 A}\left(p^{2} ; m_{1}^{2}, m_{2}^{2}, m_{3}^{2}, m_{4}^{2}\right)= & T_{1234}\left(p^{2} ; m_{1}^{2}, m_{2}^{2}, 0,0\right), \\
T_{1234 N}\left(p^{2} ; m_{1}^{2}, m_{2}^{2}, m_{3}^{2}, m_{4}^{2}\right)= & T_{1234}\left(p^{2} ; m_{1}^{2}, m_{2}^{2}, m_{3}^{2}, m_{4}^{2}\right) \\
& -T_{1234}\left(p^{2} ; m_{1}^{2}, m_{2}^{2}, 0,0\right), \\
T_{11234 A}\left(p^{2} ; m_{1}^{2}, m_{1}^{2}, m_{2}^{2}, m_{3}^{2}, m_{4}^{2}\right)= & T_{11234}\left(p^{2} ; m_{1}^{2}, m_{1}^{2}, m_{2}^{2}, 0,0\right), \\
T_{11234 N}\left(p^{2} ; m_{1}^{2}, m_{1}^{2}, m_{2}^{2}, m_{3}^{2}, m_{4}^{2}\right)= & T_{11234}\left(p^{2} ; m_{1}^{2}, m_{1}^{2}, m_{2}^{2}, m_{3}^{2}, m_{4}^{2}\right) \\
& -T_{11234}\left(p^{2} ; m_{1}^{2}, m_{1}^{2}, m_{2}^{2}, 0,0\right) .
\end{aligned}
$$

Let us briefly summarize the method used in ref. [4] to calculate the scalar integral $T_{12345}$. For timelike momenta $p$, one can work in the $p$-rest frame. Integrating over the space components of the loop momenta analytically, one is left with a two dimensional integral over the time components of the loop momenta, which can be written as

$$
\begin{aligned}
& T_{12345}\left(p^{2} ; m_{1}^{2}, m_{2}^{2}, m_{3}^{2}, m_{4}^{2}, m_{5}^{2}\right)= \\
& -\frac{4}{p^{2}} \int_{-\infty}^{\infty} \mathrm{d} x \int_{-\infty}^{\infty} \mathrm{d} y \frac{1}{w_{1}^{2}-w_{2}^{2}} \frac{1}{w_{4}^{2}-w_{5}^{2}} \ln \left(\frac{\left(w_{1}+w_{3}+w_{4}\right)\left(w_{2}+w_{3}+w_{5}\right)}{\left(w_{2}+w_{3}+w_{4}\right)\left(w_{1}+w_{3}+w_{5}\right)}\right),
\end{aligned}
$$

with

$$
w_{1}=\left[x^{2}-\frac{m_{1}^{2}}{p^{2}}+i \varepsilon\right]^{1 / 2}
$$




$$
\begin{aligned}
& w_{2}=\left[(x+1)^{2}-\frac{m_{2}^{2}}{p^{2}}+i \varepsilon\right]^{1 / 2} \\
& w_{3}=\left[(x+y)^{2}-\frac{m_{3}^{2}}{p^{2}}+i \varepsilon\right]^{1 / 2} \\
& w_{4}=\left[y^{2}-\frac{m_{4}^{2}}{p^{2}}+i \varepsilon\right]^{1 / 2} \\
& w_{5}=\left[(y-1)^{2}-\frac{m_{5}^{2}}{p^{2}}+i \varepsilon\right]^{1 / 2}:
\end{aligned}
$$

The square roots $w_{1}, \ldots, w_{5}$ should all be chosen in the first quadrant. The result, (16), is also valid for spacelike external momenta. It turns out that the remaining $x$ - and $y$-integrations can easily be performed numerically.

Following exactly the same arguments, analogous representations for the integrals $T_{N}$ defined above can be derived:

$$
\begin{aligned}
& T_{234 N}\left(p^{2} ; m_{2}^{2}, m_{3}^{2}, m_{4}^{2}\right)= \\
& \quad-4 p^{2} \int_{-\infty}^{\infty} \mathrm{d} x \int_{-\infty}^{\infty} \mathrm{d} y \ln \left(\frac{\left(w_{2}+w_{3}+w_{4}\right)\left(w_{2}+\tilde{w}_{3}+\tilde{w}_{4}\right)}{\left(w_{2}+\tilde{w}_{3}+w_{4}\right)\left(w_{2}+w_{3}+\tilde{w}_{4}\right)}\right),
\end{aligned}
$$

$$
\begin{aligned}
& T_{1234 N}\left(p^{2} ; m_{1}^{2}, m_{2}^{2}, m_{3}^{2}, m_{4}^{2}\right)= \\
& \quad 4 \int_{-\infty}^{\infty} \mathrm{d} x \int_{-\infty}^{\infty} \mathrm{d} y \frac{1}{w_{1}^{2}-w_{2}^{2}} \ln \left(\frac{\left(w_{1}+w_{3}+w_{4}\right)\left(w_{2}+\tilde{w}_{3}+\tilde{w}_{4}\right)}{\left(w_{2}+w_{3}+w_{4}\right)\left(w_{1}+\tilde{w}_{3}+\tilde{w}_{4}\right)}\right)
\end{aligned}
$$

$$
\begin{aligned}
& T_{11234 N}\left(p^{2} ; m_{1}^{2}, m_{1}^{2}, m_{2}^{2}, m_{3}^{2}, m_{4}^{2}\right)= \\
& \frac{4}{p^{2}} \int_{-\infty}^{\infty} \mathrm{d} x \int_{-\infty}^{\infty} \mathrm{d} y \frac{1}{\left(w_{1}^{2}-w_{2}^{2}\right)^{2}}\left\{\ln \left(\frac{\left(w_{1}+w_{3}+w_{4}\right)\left(w_{2}+\tilde{w}_{3}+\tilde{w}_{4}\right)}{\left(w_{2}+w_{3}+w_{4}\right)\left(w_{1}+\tilde{w}_{3}+\tilde{w}_{4}\right)}\right)\right. \\
& \left.\quad-\frac{\left(w_{1}^{2}-w_{2}^{2}\right)\left(\tilde{w}_{3}+\tilde{w}_{4}-w_{3}-w_{4}\right)}{2 w_{1}\left(w_{1}+w_{3}+w_{4}\right)\left(w_{1}+\tilde{w}_{3}+\tilde{w}_{4}\right)}\right\},
\end{aligned}
$$

where, in addition to $w_{1}, \ldots, w_{5}$, the variables

$$
\begin{aligned}
& \tilde{w}_{3}=\left[(x+y)^{2}+i \varepsilon\right]^{1 / 2}, \\
& \tilde{w}_{4}=\left[y^{2}+i \varepsilon\right]^{1 / 2}
\end{aligned}
$$

are used.

It is possible to take the limit $\varepsilon \rightarrow 0$ before performing the $x$-and $y$-integrations, provided one remembers that the square roots $w_{i}$ should be positive when $w_{i}^{2}$ is positive, and on the positive imaginary axis when $w_{i}^{2}$ is negative. When $p^{2}$ is negative, the $w_{i}$ are always positive, and the integrals $T_{N}$ are real. However, when $p^{2}$ is positive, there are regions where some of the $w_{i}$ are imaginary, giving rise to imaginary parts in the integrands. If $p^{2}$ is still below the first physical threshold of the diagram, then the integral over $x$ and $y$ of these imaginary parts vanishes. When $p^{2}$ rises above the first physical threshold, this cancellation no longer occurs and hence the integrals $T_{N}$ acquire an imaginary part. Note that the lowest threshold is determined by the diagrams that are subtracted to make the 
integrals finite. For instance, $T_{1234}\left(p^{2} ; m_{1}^{2}, m_{2}^{2}, m_{3}^{2}, m_{4}^{2}\right)$ has thresholds at $p^{2}=\left(m_{1}+m_{2}\right)^{2}$ and at $p^{2}=\left(m_{2}+m_{3}+m_{4}\right)^{2}$, but the lowest threshold of $T_{1234 N}\left(p^{2} ; m_{1}^{2}, m_{2}^{2}, m_{3}^{2}, m_{4}^{2}\right)$ is at $p^{2}=m_{2}^{2}$, because of the term $-T_{1234}\left(p^{2} ; m_{1}^{2}, m_{2}^{2}, 0,0\right)$ in its definition.

There are no singularities in the integrands in eqs. (16), (23), (24) where the denominators $w_{1}^{2}-w_{2}^{2}$ and $w_{4}^{2}-w_{5}^{2}$ vanish, since the numerators also vanish at those points. When $p^{2}>0$, the integrand of eq. (24) is singular at the points where $w_{1}=0$. As long as $m_{1} \neq 0$, these are integrable singularities.

In order to perform the numerical integrations, we map the integration region onto a unit square. For $T_{1234 N}$ and $T_{11234 N}$ in the case when $p^{2}<0$, we first shift the integration variables so that the zero of the denominator $w_{1}^{2}-w_{2}^{2}$ coincides with the $y$-axis. Then, we map the infinite plane onto one quadrant:

$$
\begin{aligned}
& \int_{-\infty}^{\infty} \mathrm{d} x \int_{-\infty}^{\infty} \mathrm{d} y F(x, y)= \\
& \quad \int_{0}^{\infty} \mathrm{d} x \int_{0}^{\infty} \mathrm{d} y\{F(x, y)+F(-x, y)+F(x,-y)+F(-x,-y)\} .
\end{aligned}
$$

Finally, we map this quadrant onto a unit square using $x=x^{\prime} /\left(1-x^{\prime}\right)$ and $y=y^{\prime} /\left(1-y^{\prime}\right)$. We use the Monte Carlo program VEGAS [13] to integrate over $x^{\prime}$ and $y^{\prime}$.

More care is needed for $T_{11234 N}$ in the case when $p^{2}>0$. As in the other cases, we map the infinite plane onto one quadrant, but we do not perform the shift. Instead, we split the $x$-integral into two parts: one from 0 to $x_{0} \equiv m_{1} / \sqrt{p^{2}}$ and one from $x_{0}$ to $\infty$. In the former we use the transformation $x=x_{0}\left(1-x^{2}\right) /\left(1+x^{2}\right)$, in the latter $x=x_{0}\left(1+x^{2}\right) /\left(1-x^{\prime 2}\right)$. In this way, the Jacobians cancel the troublesome factor $w_{1}$ in the denominator of (24).

\section{Comparisons}

To check the reliability of the two-dimensional integration method, we compared it with results obtained by other methods. These are:

1. Known analytical results for cases where one or more masses vanish.

2. In one case the imaginary part of a diagram is evaluated by a one-dimensional numerical integration.

3. Small $p^{2}$ expansions [5], convergent when $\left|p^{2}\right|$ is smaller than the lowest threshold of the diagram.

4. Large $p^{2}$ expansions [6] that converge when $\left|p^{2}\right|$ is larger than the highest threshold of the diagram.

For all three diagrams comparisons with the above types of results were made. The outcome will be discussed below, and a few examples will be explicitly shown in tables. We can define a relative statistical error in the numerical integration as the quotient of the standard deviation and the average result as reported by VEGAS. We use two iterations of 20000 points to adjust the grid, and then one iteration with 100000 points for the final integration. This typically gives a relative statistical error of $10^{-4}$, but for some values of the masses and momenta, it is larger. This is an indication of trouble with the numerical integration. In every instance where a serious discrepancy between the numerical integration another method was found, the relative statistical error was greater than $10^{-3}$. 


\section{Tests of $T_{234 N}$.}

1.1 The case $m_{2}=0$, is compared with the analytical result (38). For $m_{3}=5$, $m_{4}=7$ and $-1000<p^{2}<1000$, the agreement is generally good (four digits). The agreement is less good in the imaginary part close to $p^{2}=0$, due to the cancellations mentioned above.

1.2 For general values of the masses, the following one-dimensional integral representation can be obtained from Cutkosky's rules [12]:

$$
\begin{aligned}
\operatorname{Im} & T_{234}\left(p^{2} ; m_{2}^{2}, m_{3}^{2}, m_{4}^{2}\right) \\
& =-\frac{\pi}{p^{2}} \int_{\left(m_{3}+m_{4}\right)^{2}}^{\left(\sqrt{p^{2}}-m_{2}\right)^{2}} \mathrm{~d} s \frac{1}{s} \sqrt{\lambda\left(p^{2}, s, m_{2}^{2}\right) \lambda\left(s, m_{3}^{2}, m_{4}^{2}\right)},
\end{aligned}
$$

where $\lambda$ is the Källén function:

$$
\lambda(a, b, c)=(a-b-c)^{2}-4 b c .
$$

A comparison is shown in table 1 .

1.3 Agreement to three digits with the small $p^{2}$ expansion is found for $m_{2}=3$, $m_{3}=5$ and $m_{4}=7$ and $p^{2}$ in the range $0.004<p^{2}<9$.

1.4 Three to four digit agreement with the large $p^{2}$ expansion is found for $m_{2}=3$, $m_{3}=5$ and $m_{4}=7$ and $p^{2}$ in the range $500<p^{2}<3 \times 10^{5}$.

2. Tests of $T_{1234 N}$.

2.1 Three special mass cases are checked. The first is when $m_{1}=m_{2}=m_{4}=0$ and $m_{3}=m$, given analytically in [9], and the second is $m_{1}=m_{2}=0$ and $m_{3}=m_{4}=m$, given by eq. (40). With $m=1$, three to four digit agreement is found for $-1000<p^{2}<-0.1$ and $0.1<p^{2}<1000$. The third case is $m_{1}=m_{4}$, $m_{3}=0$, which can be found in reference [11]. A comparison is shown in table 2 . Again, the effect of cancellations in the imaginary part just above the threshold can be seen; otherwise, there is good agreement.

2.2 A comparison with the small $p^{2}$ expansion is shown in table 3 .

2.3 Three to four digit agreement with the large $p^{2}$ expansion is found for $m_{1}=1$, $m_{2}=3, m_{3}=5$ and $m_{4}=7$ and $p^{2}$ in the range $500<p^{2}<3 \times 10^{5}$.

3. Tests of $T_{11234 N}$.

3.1 The special case $m_{1}=m_{4}, m_{3}=0$, given in reference [11], is used here as a check. With $m_{1}=1$ and $m_{2}=2$, there is four digit agreement for $-1000<p^{2}<$ -0.01 . For positive $p^{2}$, the results are shown in table 4. At the pseudothreshold $p^{2}=\left(m_{2}-m_{1}\right)^{2}$ and the threshold $p^{2}=\left(m_{2}+m_{1}\right)^{2}$, the representation (24) is singular because $w_{1}$ and $w_{2}$ both vanish at the same point. The integral $T_{11234 N}$ itself is, indeed, divergent on the threshold. The origin of this divergence is the region in momentum space where the propagator with momentum $k_{2}$ and the two propagators with momentum $k_{1}$ are on the mass shell. Nevertheless, the results in the vicinity of these (pseudo)thresholds are reasonable.

3.2 With the masses $m_{1}=1, m_{2}=3, m_{3}=5$ and $m_{4}=7$, the numerical integration result agrees with the small $p^{2}$ expansion to three digits for both positive and negative $p^{2}$ in the range $0.004<\left|p^{2}\right|<2$. 
3.3 Table 5 shows a comparison with the large $p^{2}$ expansion for positive $p^{2}$. The agreement for the real part is good, but not for the imaginary part. For negative $p^{2}$ in the range $-5 \times 10^{5}<p^{2}<-225$ and the same masses there is four to five digit agreement.

\begin{tabular}{|c|c|c|c|}
\hline$p^{2}$ & $\mathrm{~A}$ & $\mathrm{~B}$ & $\mathrm{C}$ \\
\hline 15.0000 & -0.0417649 & $0.21 \times 10^{-1}$ & -0.042426 \\
30.0000 & -0.2942085 & $0.13 \times 10^{-2}$ & -0.294707 \\
45.0000 & -0.4967213 & $0.49 \times 10^{-3}$ & -0.496725 \\
60.0000 & -0.6411420 & $0.27 \times 10^{-3}$ & -0.641457 \\
75.0000 & -0.7418943 & $0.17 \times 10^{-3}$ & -0.741856 \\
90.0000 & -0.7915522 & $0.13 \times 10^{-3}$ & -0.791607 \\
105.000 & -0.8086538 & $0.11 \times 10^{-3}$ & -0.808604 \\
120.000 & -0.7991393 & $0.87 \times 10^{-4}$ & -0.799156 \\
135.000 & -0.7691024 & $0.77 \times 10^{-4}$ & -0.769065 \\
150.000 & -0.7264257 & $0.70 \times 10^{-4}$ & -0.726384 \\
165.000 & -0.6766883 & $0.67 \times 10^{-4}$ & -0.676697 \\
180.000 & -0.6235912 & $0.63 \times 10^{-4}$ & -0.62357 \\
195.000 & -0.5692483 & $0.61 \times 10^{-4}$ & -0.569249 \\
210.000 & -0.5151574 & $0.61 \times 10^{-4}$ & -0.515106 \\
225.000 & -0.4619533 & $0.59 \times 10^{-4}$ & -0.461988 \\
240.000 & -0.4138414 & $0.56 \times 10^{-4}$ & -0.413862 \\
\hline
\end{tabular}

Table 1: The imaginary part of $\frac{1}{p^{2}} T_{234 N}\left(p^{2} ; m_{2}^{2}, m_{3}^{2}, m_{4}^{2}\right)$, with $m_{2}=3, m_{3}=5$ and $m_{4}=7$. A: result of integration of (22) using VEGAS. B: relative error reported by VEGAS. C: result obtained from (28). 


\begin{tabular}{|c|c|c|c|c|c|c|}
\hline & \multicolumn{3}{|c|}{ Real part } & \multicolumn{3}{|c|}{ Imaginary part } \\
\hline$p^{2}$ & $\mathrm{~A}$ & $\mathrm{~B}$ & $\mathrm{C}$ & $\mathrm{A}$ & $\mathrm{B}$ & $\mathrm{C}$ \\
\hline-1000 & -0.03874178 & $0.48 \times 10^{-4}$ & -0.0387435 & & & \\
-100 & -0.1997168 & $0.36 \times 10^{-4}$ & -0.199719 & & & \\
-10.0 & -0.6285239 & $0.28 \times 10^{-4}$ & -0.628509 & & & \\
-1.0 & -0.9267035 & $0.34 \times 10^{-4}$ & -0.926689 & & & \\
-0.1 & -0.9802995 & $0.57 \times 10^{-4}$ & -0.98029 & & & \\
-0.01 & -0.9866642 & $0.37 \times 10^{-3}$ & -0.986114 & & & \\
0.01 & -0.9883377 & $0.52 \times 10^{-2}$ & -0.987421 & & & \\
0.1 & -0.9934639 & $0.48 \times 10^{-3}$ & -0.993359 & & & \\
1.0 & -1.056218 & $0.16 \times 10^{-2}$ & -1.05855 & & & \\
2.0 & -1.146370 & $0.18 \times 10^{-3}$ & -1.14664 & & & \\
4.00 & -1.412433 & $0.13 \times 10^{-3}$ & -1.41219 & 0.00003568 & $0.53 \times 10^{1}$ & 0 \\
4.1 & -1.431185 & $0.13 \times 10^{-3}$ & -1.43126 & -0.0000108 & $0.19 \times 10^{2}$ & -0.00003154 \\
4.2 & -1.451126 & $0.12 \times 10^{-3}$ & -1.4514 & -0.0001076 & $0.18 \times 10^{1}$ & -0.00024351 \\
4.4 & -1.495182 & $0.12 \times 10^{-3}$ & -1.49503 & -0.0018140 & 0.10 & -0.0018211 \\
4.6 & -1.542992 & $0.11 \times 10^{-3}$ & -1.54307 & -0.0056238 & $0.32 \times 10^{-1}$ & -0.0057718 \\
4.8 & -1.595209 & $0.10 \times 10^{-3}$ & -1.5953 & -0.0129236 & $0.14 \times 10^{-1}$ & -0.0129036 \\
5.0 & -1.651756 & $0.98 \times 10^{-4}$ & -1.65145 & -0.0238376 & $0.78 \times 10^{-2}$ & -0.0238659 \\
7.0 & -2.375996 & $0.66 \times 10^{-4}$ & -2.3761 & -0.4559457 & $0.39 \times 10^{-3}$ & -0.455663 \\
9.00 & -3.375098 & $0.19 \times 10^{-2}$ & -3.3758 & -3.441894 & $0.75 \times 10^{-3}$ & -3.44372 \\
10.0 & -1.051435 & $0.15 \times 10^{-3}$ & -1.05161 & -2.865103 & $0.56 \times 10^{-4}$ & -2.86505 \\
100 & 0.1687759 & $0.11 \times 10^{-3}$ & 0.168789 & -0.2262556 & $0.57 \times 10^{-4}$ & -0.226246 \\
1000 & 0.03432286 & $0.11 \times 10^{-3}$ & 0.0343225 & -0.0282523 & $0.79 \times 10^{-4}$ & -0.0282523 \\
\hline
\end{tabular}

Table 2: $T_{1234 N}\left(p^{2} ; m_{1}^{2}, m_{2}^{2}, 0, m_{1}^{2}\right)$ with $m_{1}=1$ and $m_{2}=2$. A: Result from VEGAS integration of eq. (23). B: Relative error reported by VEGAS. C: Analytical result.

\begin{tabular}{|c|c|c|c|}
\hline$p^{2}$ & $\mathrm{~A}$ & $\mathrm{~B}$ & $\mathrm{C}$ \\
\hline $\mathbf{9 . 0 0 0 0 0}$ & -10.11861 & $0.17 \times 10^{-3}$ & -9.821787 \\
4.00000 & -8.710491 & $0.61 \times 10^{-3}$ & -8.700318 \\
1.77778 & -8.286016 & $0.24 \times 10^{-3}$ & -8.286116 \\
0.790124 & -8.116017 & $0.37 \times 10^{-3}$ & -8.118665 \\
$\mathbf{0 . 3 5 1 1 6 6}$ & -8.053491 & $0.40 \times 10^{-3}$ & -8.047528 \\
$\mathbf{0 . 1 5 6 0 7 4}$ & -8.013727 & $0.39 \times 10^{-3}$ & -8.016561 \\
$\mathbf{0 . 0 6 9 3 6 6}$ & -7.999787 & $0.69 \times 10^{-3}$ & -8.002926 \\
$\mathbf{0 . 0 3 0 8 2 9}$ & -7.985865 & $0.24 \times 10^{-2}$ & -7.996891 \\
$\mathbf{0 . 0 1 3 7 0 2}$ & -7.900682 & $\mathbf{0 . 4 4} \times 10^{-2}$ & -7.994214 \\
$\mathbf{0 . 0 0 6 0 9 0}$ & -7.793835 & $0.31 \times 10^{-1}$ & -7.993026 \\
\hline
\end{tabular}

Table 3: Real part of $T_{1234 N}\left(p^{2} ; m_{1}^{2}, m_{2}^{2}, m_{3}^{2}, m_{4}^{2}\right)$ for small values of $p^{2}$. The masses are $m_{1}=1$, $m_{2}=3, m_{3}=5$ and $m_{4}=7$. A: Result from VEGAS integration of eq. (23). B: Relative error reported by VEGAS. C: Approximation using 3 terms of Taylor expansion. 


\begin{tabular}{|c|c|c|c|c|c|c|}
\hline & \multicolumn{3}{|c|}{ Real part } & \multicolumn{3}{|c|}{ Imaginary part } \\
\hline$p^{2}$ & $\mathrm{~A}$ & $\mathrm{~B}$ & $\mathrm{C}$ & $\mathrm{A}$ & $\mathrm{B}$ & $\mathrm{C}$ \\
\hline 0.01 & 0.0021980 & $0.98 \times 10^{-3}$ & 0.0021967 & & & \\
0.10 & 0.0222252 & $0.11 \times 10^{-2}$ & 0.0222253 & & & \\
0.90 & 0.2245398 & $0.55 \times 10^{-2}$ & 0.2233319 & & & \\
0.99 & 0.2477748 & $0.15 \times 10^{-1}$ & 0.248925 & & & \\
1.00 & $0.57 \times 10^{7}$ & 0.61 & 0.251811 & & & \\
1.01 & 0.2462982 & $0.18 \times 10^{-1}$ & 0.254705 & & & \\
1.10 & 0.2790692 & $0.60 \times 10^{-2}$ & 0.281143 & & & \\
2.00 & 0.5904654 & $0.52 \times 10^{-3}$ & 0.590847 & & & \\
3.00 & 1.072371 & $0.16 \times 10^{-3}$ & 1.07245 & & & \\
4.00 & 1.819090 & $0.17 \times 10^{-3}$ & 1.81893 & & & \\
4.10 & 1.918535 & $0.15 \times 10^{-3}$ & 1.91868 & 0.0002918 & $0.15 \times 10^{1}$ & 0.0001293 \\
4.20 & 2.025207 & $0.17 \times 10^{-3}$ & 2.02548 & 0.0007309 & 0.43 & 0.0010237 \\
4.40 & 2.262846 & $0.28 \times 10^{-3}$ & 2.26233 & 0.0077592 & $0.47 \times 10^{-1}$ & 0.0080436 \\
4.60 & 2.531991 & $0.14 \times 10^{-3}$ & 2.5326 & 0.0268762 & $0.18 \times 10^{-1}$ & 0.0267768 \\
4.80 & 2.838831 & $0.19 \times 10^{-3}$ & 2.83868 & 0.0627915 & $0.99 \times 10^{-2}$ & 0.0628651 \\
5.00 & 3.184130 & $0.22 \times 10^{-3}$ & 3.18272 & 0.1219940 & $0.44 \times 10^{-2}$ & 0.122104 \\
7.00 & 9.380864 & $0.15 \times 10^{-3}$ & 9.38079 & 3.974197 & $0.52 \times 10^{-3}$ & 3.97556 \\
8.00 & 15.90483 & $0.20 \times 10^{-3}$ & 15.9085 & 13.75989 & $0.23 \times 10^{-3}$ & 13.7594 \\
8.90 & 31.89093 & $0.69 \times 10^{-3}$ & 31.9269 & 102.2986 & $0.20 \times 10^{-3}$ & 102.288 \\
8.99 & 40.10836 & $0.35 \times 10^{-2}$ & 40.2817 & 388.6728 & $0.39 \times 10^{-3}$ & 388.532 \\
8.999 & 43.66791 & $0.18 \times 10^{-1}$ & 44.1650 & 1294.293 & $0.11 \times 10^{-2}$ & 1293.98 \\
9.00 & $-0.21 \times 10^{9}$ & 0.53 & $*$ & $0.18 \times 10^{9}$ & 0.70 & $*$ \\
9.001 & -1277.272 & $0.59 \times 10^{-3}$ & -1278.17 & -27.53089 & $0.40 \times 10^{-1}$ & -27.4416 \\
9.01 & -374.5440 & $0.36 \times 10^{-3}$ & -374.635 & -23.77220 & $0.74 \times 10^{-2}$ & -23.6635 \\
10.0 & -15.54301 & $0.22 \times 10^{-3}$ & -15.5495 & -6.016426 & $0.53 \times 10^{-3}$ & -6.02052 \\
100 & -1.747562 & $0.39 \times 10^{-3}$ & -1.7469 & -0.001904 & 0.21 & -0.002749 \\
1000 & -1.656141 & $0.41 \times 10^{-3}$ & -1.65607 & 0.0009338 & 0.60 & 0.0013192 \\
\hline
\end{tabular}

Table 4: $p^{2} T_{11234 N}\left(p^{2} ; m_{1}^{2}, m_{1}^{2}, m_{2}^{2}, 0, m_{1}^{2}\right)$ for positive $p^{2}$. The masses are $m_{1}=1$ and $m_{2}=2$. A: Result from VEGAS integration of eq. (24). B: Relative error reported by VEGAS. C: Analytical result. * At the threshold $p^{2}=\left(m_{1}+m_{2}\right)^{2}=9$, the integral diverges. 


\begin{tabular}{|c|c|c|c|c|c|c|}
\hline & \multicolumn{3}{|c|}{ Real part } & \multicolumn{3}{c|}{ Imaginary part } \\
\hline$p^{2}$ & $\mathrm{~A}$ & $\mathrm{~B}$ & $\mathrm{C}$ & $\mathrm{A}$ & $\mathrm{B}$ & $\mathrm{C}$ \\
\hline 225.000 & -15.15159 & $0.88 \times 10^{-4}$ & -15.09431 & 0.1773819 & $0.82 \times 10^{-2}$ & 0.1955739 \\
506.250 & -14.27563 & $0.11 \times 10^{-3}$ & -14.27394 & 0.1463133 & $0.11 \times 10^{-1}$ & 0.1493184 \\
1139.06 & -13.94513 & $0.12 \times 10^{-3}$ & -13.94187 & 0.0815159 & $0.22 \times 10^{-1}$ & 0.0827948 \\
2562.89 & -13.78969 & $0.13 \times 10^{-3}$ & -13.78785 & 0.0368462 & $0.53 \times 10^{-1}$ & 0.0408465 \\
5766.50 & -13.71204 & $0.15 \times 10^{-3}$ & -13.71368 & 0.0209738 & 0.12 & 0.0191222 \\
12974.6 & -13.67441 & $0.14 \times 10^{-3}$ & -13.67780 & 0.0123064 & 0.21 & 0.0087250 \\
29192.9 & -13.65539 & $0.18 \times 10^{-3}$ & -13.66053 & 0.0039702 & 0.64 & 0.0039296 \\
65684.1 & -13.65113 & $0.16 \times 10^{-3}$ & -13.65226 & 0.0020849 & $0.11 \times 10^{1}$ & 0.0017582 \\
147789. & -13.64576 & $0.20 \times 10^{-3}$ & -13.64833 & -0.0013321 & $0.22 \times 10^{1}$ & 0.0007840 \\
332526. & -13.64775 & $0.21 \times 10^{-3}$ & -13.64646 & 0.0026535 & $0.26 \times 10^{1}$ & 0.0003490 \\
\hline
\end{tabular}

Table 5: $p^{2} T_{11234 N}\left(p^{2} ; m_{1}^{2}, m_{1}^{2}, m_{2}^{2}, m_{3}^{2}, m_{4}^{2}\right)$ for large positive values of $p^{2}$. The masses are $m_{1}=$ $1, m_{2}=3, m_{3}=5$ and $m_{4}=7$. A: Result from VEGAS integration of eq. (24). B: Relative error reported by VEGAS. C: Approximation using asymptotic expansion for $p^{2} \rightarrow \infty$. The first neglected term is of order $\ln ^{2}\left(p^{2}\right) /\left(p^{2}\right)^{4}$.

\section{Conclusions}

Applying the method of reference [4], we have obtained two-dimensional integral representations for all the scalar two-loop diagrams that are required for the calculation of Standard Model gauge boson self-energies. The results were checked in several special cases where other methods of computing them were available. For a wide range of parameter values, there is three to four digit agreement. Whenever the results do not agree, we are warned by the large relative statistical error.

The great advantage of this method is its generality. In special cases, it cannot compete in speed or precision with analytical formulae, but in the general mass case, it provides results where no other methods are available. Another advantage is its simplicity. In principle, it is possible to derive one dimensional integral representations for these scalar diagrams, but one would have to consider different cases, depending on whether some masses are larger or smaller than others, separately. With Kreimer's method, all cases are treated at the same time.

\section{Appendix}

In this appendix, we give analytical formulae for a number of special cases. They are expanded up to $\mathcal{O}(1)$ in $\delta$, where $D=4-2 \delta$ is the dimension of space-time. We define the logarithm $\ln (z)$ with a cut along the the negative real axis, and the dilogarithm $\operatorname{Li}_{2}(z)$ with a cut along the positive real axis for $z>1$ :

$$
\operatorname{Li}_{2}(z)=-\int_{0}^{1} \mathrm{~d} t \frac{\ln (1-z t)}{t}
$$


We use the abbreviations

$$
L_{p}=\gamma_{E}+\ln \left(\frac{-p^{2}}{4 \pi \mu^{2}}\right), L_{m_{i}}=\gamma_{E}+\ln \left(\frac{m_{i}^{2}}{4 \pi \mu^{2}}\right)
$$

where $\gamma_{E}$ is Euler's constant. $\zeta$ is the Riemann zeta function.

The vacuum integrals $T_{134}$ and $T_{1134}$. See also refs. [5, 10, 14].

$$
\begin{aligned}
& T_{134}\left(p^{2} ; m_{1}^{2}, m_{2}^{2}, m_{3}^{2}\right)= \\
& \quad \frac{1}{2 \delta^{2}}\left(m_{1}^{2}+m_{2}^{2}+m_{3}^{2}\right) \\
& +\frac{1}{\delta}\left\{\frac{3}{2}\left(m_{1}^{2}+m_{2}^{2}+m_{3}^{2}\right)-m_{1}^{2} L_{m_{1}}-m_{2}^{2} L_{m_{2}}-m_{3}^{2} L_{m_{3}}\right\} \\
& +\left(\frac{7}{2}+\frac{1}{2} \zeta(2)\right)\left(m_{1}^{2}+m_{2}^{2}+m_{3}^{2}\right) \\
& +m_{1}^{2}\left(L_{m_{1}}^{2}-3 L_{m_{1}}\right)+m_{2}^{2}\left(L_{m_{2}}^{2}-3 L_{m_{2}}\right)+m_{3}^{2}\left(L_{m_{3}}^{2}-3 L_{m_{3}}\right) \\
& +\frac{1}{4}\left(m_{1}^{2}-m_{2}^{2}-m_{3}^{2}\right) \ln ^{2}\left(\frac{m_{2}^{2}}{m_{3}^{2}}\right)+\frac{1}{4}\left(-m_{1}^{2}+m_{2}^{2}-m_{3}^{2}\right) \ln ^{2}\left(\frac{m_{1}^{2}}{m_{3}^{2}}\right) \\
& +\frac{1}{4}\left(-m_{1}^{2}-m_{2}^{2}+m_{3}^{2}\right) \ln ^{2}\left(\frac{m_{1}^{2}}{m_{2}^{2}}\right)+\frac{1}{2} F\left(m_{1}^{2}, m_{2}^{2}, m_{3}^{2}\right) \\
& \quad T_{1134}\left(p^{2} ; m_{1}^{2}, m_{1}^{2}, m_{2}^{2}, m_{3}^{2}\right)= \\
& \quad \frac{1}{2 \delta^{2}}+\frac{1}{\delta}\left(\frac{1}{2}-L_{m_{1}}\right)+\frac{1}{2}+\frac{1}{2} \zeta(2)+L_{m_{1}}^{2}-L_{m_{1}} \\
& \quad+\frac{1}{4} \ln ^{2}\left(\frac{m_{2}^{2}}{m_{3}^{2}}\right)-\frac{1}{4} \ln ^{2}\left(\frac{m_{1}^{2}}{m_{2}^{2}}\right)-\frac{1}{4} \ln ^{2}\left(\frac{m_{1}^{2}}{m_{3}^{2}}\right) \\
& \quad+\frac{1}{2} \frac{m_{1}^{2}-m_{2}^{2}-m_{3}^{2}}{\lambda\left(m_{1}^{2}, m_{2}^{2}, m_{3}^{2}\right)} F\left(m_{1}^{2}, m_{2}^{2}, m_{3}^{2}\right)
\end{aligned}
$$

The function $F$ is symmetric with respect to $m_{1}^{2}, m_{2}^{2}$ and $m_{3}^{2}$, and can be written as

$$
F\left(m_{1}^{2}, m_{2}^{2}, m_{3}^{2}\right)=m_{3}^{2} \lambda^{2}(x, y) \Phi(x, y)
$$

with

$$
\begin{gathered}
x \equiv \frac{m_{1}^{2}}{m_{3}^{2}}, \quad y \equiv \frac{m_{2}^{2}}{m_{3}^{2}}, \\
\lambda^{2}(x, y)=(1-x-y)^{2}-4 x y, \\
\Phi(x, y)=\frac{1}{\lambda}\left\{2 \ln \left(\frac{1+x-y-\lambda}{2}\right) \ln \left(\frac{1-x+y-\lambda}{2}\right)-\ln x \ln y\right. \\
\left.-2 \operatorname{Li}_{2}\left(\frac{1+x-y-\lambda}{2}\right)-2 \operatorname{Li}_{2}\left(\frac{1-x+y-\lambda}{2}\right)+\frac{\pi^{2}}{3}\right\} .
\end{gathered}
$$

If the largest mass is $m_{1}$ or $m_{2}$, we should choose this mass as the dimensionless-making parameter in (34) and (35) (instead of $m_{3}$ ). 
When using eqs. (38)-(41), $p^{2}$ should be given an infinitesimal positive imaginary part. The integral $T_{234}$, when one of the masses is zero.

$$
\begin{aligned}
T_{234} & \left(p^{2} ; m_{1}^{2}, m_{2}^{2}, 0\right)= \\
& \frac{1}{2 \delta^{2}}\left(m_{1}^{2}+m_{2}^{2}\right) \\
& \frac{1}{\delta}\left\{\frac{3}{2}\left(m_{1}^{2}+m_{2}^{2}\right)-m_{1}^{2} L_{m_{1}}-m_{2}^{2} L_{m_{2}}-\frac{1}{4} p^{2}\right\} \\
& +m_{1}^{2}\left(L_{m_{1}}^{2}-3 L_{m_{1}}\right)+m_{2}^{2}\left(L_{m_{2}}^{2}-3 L_{m_{2}}\right)+\frac{1}{2} p^{2} L_{p} \\
& +\frac{1}{4} p^{2}\left\{\ln \left(\frac{m_{1}^{2}}{-p^{2}}\right)+\ln \left(\frac{m_{2}^{2}}{-p^{2}}\right)-\frac{13}{2}\right\} \\
& +\left(m_{1}^{2}+m_{2}^{2}\right)\left\{3+\frac{1}{2} \zeta(2)-\frac{1}{4} \ln ^{2}\left(\frac{m_{1}^{2}}{m_{2}^{2}}\right)\right\} \\
& +\frac{1}{2}\left(m_{1}^{2}-m_{2}^{2}\right)\left\{\operatorname{Li}_{2}\left(\frac{m_{1}^{2}-m_{2}^{2}}{m_{1}^{2}}\right)-\mathrm{Li}_{2}\left(\frac{m_{2}^{2}-m_{1}^{2}}{m_{2}^{2}}\right)\right\} \\
& +\frac{1}{4} p^{2}\left\{\left(\frac{m_{1}^{2}}{p^{2}}\right)-\left(\frac{m_{2}^{2}}{p^{2}}\right)^{2}\right\} \ln \left(\frac{m_{1}^{2}}{m_{2}^{2}}\right) \\
& +\frac{1}{4}\left(p^{2}+m_{1}^{2}+m_{2}^{2}\right) \frac{m_{2}^{2}}{p^{2}}\left(r_{1}-r_{2}\right)\left\{-\ln \left(r_{1}\right)+\ln \left(r_{2}\right)\right\} \\
& +m_{1}^{2}\left(1-\frac{m_{2}^{2}}{p^{2}}\right)\left\{\operatorname{Li}_{2}\left(\frac{1-r_{1}}{-r_{1}}\right)+\mathrm{Li}_{2}\left(\frac{1-r_{2}}{-r_{2}}\right)-\mathrm{Li}_{2}\left(\frac{m_{1}^{2}-m_{2}^{2}}{m_{1}^{2}}\right)\right\} \\
& +m_{2}^{2}\left(1-\frac{m_{1}^{2}}{p^{2}}\right)\left\{\operatorname{Li}_{2}\left(1-r_{1}\right)+\operatorname{Li}_{2}\left(1-r_{2}\right)-\mathrm{Li}_{2}\left(\frac{m_{2}^{2}-m_{1}^{2}}{m_{2}^{2}}\right)\right\}
\end{aligned}
$$

where $r_{1}$ and $r_{2}$ are the roots of the equation

$$
m_{2}^{2} r+\frac{m_{1}^{2}}{r}=m_{1}^{2}+m_{2}^{2}-p^{2} .
$$

A special case of $T_{1234}$.

$$
\begin{aligned}
& T_{1234}\left(p^{2} ; 0,0, m^{2}, m^{2}\right)= \\
& \frac{1}{2 \delta^{2}}+\frac{1}{\delta}\left\{\frac{5}{2}-L_{p}\right\}+\frac{19}{2}-\frac{1}{2} \zeta(2)+L_{p}^{2}-5 L_{p} \\
& -\frac{1}{2} \ln ^{2}\left(\frac{-p^{2}}{m^{2}}\right)+3 \ln \left(\frac{-p^{2}}{m^{2}}\right) \\
& +3 \frac{m^{2}}{p^{2}}\left(r_{1}-r_{2}\right) \ln \left(r_{1}\right)+\frac{1}{2}\left(1+\frac{2 m^{2}}{p^{2}}\right) \ln ^{2}\left(r_{1}\right),
\end{aligned}
$$

where $r_{1}$ and $r_{2}$ are the roots of the equation

$$
m^{2} r+\frac{m^{2}}{r}=2 m^{2}-p^{2} .
$$




\section{References}

[1] F.A. Berends, G. Burgers and W.L. van Neerven, Nucl. Phys. B297 (1988) 429; erratum: B304 (1988) 921; D.Yu. Bardin et al. in "Z physics at LEP 1", CERN 89-08, (1989) p.89; W.Beenakker, F.A. Berends and S.C. van der Marck, Nucl. Phys. B349 (1991) 323; Nucl. Phys. B367 (1991) 287.

[2] J. van der Bij and F. Hoogeveen, Nucl. Phys. B283 (1987) 477; M. Consoli, W. Hollik and F. Jegerlehner, Phys. Lett. B227 (1989) 167; R. Barbieri, M. Beccaria, P. Ciafaloni, G. Curci and A. Viceré, Phys. Lett. B288 (1992) 95; R. Barbieri, M. Beccaria, P. Ciafaloni, G. Curci and A. Viceré, Nucl. Phys. B409 (1993) 105.

[3] G. Weiglein, R. Mertig, R. Scharf and M. Böhm, in "New Computing Techniques in Physics Research II" , ed. D. Perret-Gallix, World Scientific 1992, p.617 ; G. Weiglein, R. Scharf and M. Böhm, Reduction of general two-loop self-energies to standard scalar integrals, to be published in Nucl.Phys. B.

[4] D. Kreimer, Phys.Lett. B273 (1991) 277.

[5] A.I. Davydychev and J.B. Tausk, Nucl.Phys. B397 (1993) 123;

[6] A.I. Davydychev, V.A. Smirnov and J.B. Tausk, Nucl.Phys. B410 (1993) 325.

[7] D.J. Broadhurst, Z.Phys. C47 (1990) 115; D.J. Broadhurst, J. Fleischer and O.V. Tarasov, Z. Phys. C60 (1993) 287.

[8] F.A. Berends, M. Böhm, M. Buza and R. Scharf, to be published.

[9] R. Scharf, J.B. Tausk, University of Leiden preprint INLO-PUB-7/93 (1993), to be published in Nucl.Phys. B.

[10] R.Scharf, Diploma Thesis, University of Würzburg, 1991.

[11] A. Djouadi, Nuovo Cim. 100A (1988) 357;

S.C. Generalis, Open University thesis OUT-4102-13, Milton Keynes, 1984.

[12] R.E. Cutkosky, J. Math. Phys. 1 (1960) 429;

M. Veltman, Physica 29 (1963) 186;

G. 't Hooft and M. Veltman, Diagrammar, CERN Yellow Report 73-9.

[13] G.P. Lepage, J. Comp. Phys. 27 (1978) 192;

G.P. Lepage, Cornell preprint CLNS-80/447 (1980).

[14] J. van der Bij and M. Veltman, Nucl.Phys. B231 (1984) 205; F. Hoogeveen, Nucl.Phys. B259 (1985) 19; C. Ford and D.R.T. Jones, Phys.Lett. B274 (1992) 409; errata: B285 (1992) 399; C. Ford, I. Jack and D.R.T. Jones, Nucl.Phys. B387 (1992) 373. 
